\title{
Mobile Multimedia Information Management Platform
}

\author{
Wu Yu-lan, Zhang Xiao-bao \\ Nanchang University College of Science and Technology
}

\begin{abstract}
People nowadays ask higher requirement on timeliness of information, and it is not enough to get information in a fixed place while sky rocketing people expect to get important information anytime and anywhere. The emergence of mobile information processing or mobile computing aims to achieve this goal. In order to better implement government service and supervision efficiency, mobile communication can make government affairs public and serve public. This paper develops mobile multimedia information management platform, which takes advantage of the SMS/MMS technology and HTML technology, and adopts virtual technology to construct a platform for big data analysis so as to provide customers with SMS/MMS and media data service system inside the government and facing public.
\end{abstract}

KeyWords-Mobile Communication; Multimedia; Computer Application; Information processing

\section{INTRODUCTION}

The accessing to internet anywhere and anytime can maximize the information value while for consumers, the mobile information acquisition cannot be separated from network access and terminal equipment. The newest 802.11n of WLAN has improved the transmission bandwidth to $110 \mathrm{Mbit} / \mathrm{s}$, however, with the improvement of mobile communication bandwidth, Wireless LAN technology is now in an awkward position. Concerning basic information, several Mbit/s bandwidth is enough for people to acquire valuable information while hundred egabytes provided by wireless local area networks have little significance because the communication speed of wireless networks is never faster than cables.

The more important point is that, if we vividly describe user experience, WLAN is mobile office location and mobile information processing terminals can be changed from one place to another which cannot realize mobile information acquisition or at least in most transportation vehicles, WLAN cannot provide service. This is an embarrassing problem for business men who want to acquire information during travel; on the other hand, WLAN's coverage area is small, which can never include beautiful scenery and vast mountains. Therefore, for those who want to be close to the nature, they cannot expect WLAN to provide service here.

This paper deeply analyzes the mobile multimedia information service system, which is made up of short message service subsystem, MMS application subsystem, electronic certificate business application subsystem, streaming media service subsystem, and routing \& switching center system.

\section{MOBILE SHORT MESSAGE PROCESSING}

There are three data formats to send and receive message, which are Block Mode, Text Mode based on AT order and PDU Mode based on AT order. At present, PDU Mode is widely used and almost all electronic communications agencies all over the nation supports the short message business of PDU Mode. In order to ensure that the system has a wide range of applicability, this chapter mainly discusses on the PDU mode delivery which adopts 3 types of codes in receiving and sending messages, including 7-bit, 8-bit and UCS2 code. 7-bit is mainly used to deliver common ASCII characters while 8-bit is adopted to send data messages, and UCS2 code can send Unicode characters containing Chinese characters, besides, traffic information includes letters, numbers, and Chinese. When UCS2 carries out code, the user information field of PDU mode can send the maximum 70 letters and messages above 70 letters are called long messages. At present, to send and receive long message has become a basic requirement for 
mobile phones. If the traffic information message has more than 70 letters, the mobile phone shall send messages based on sequential encoding. For receivers, they identify whether they receive long message or not based on the basic parameter 51 of PDU code and the main body of TP-UD has a protocol header flag with 6 or 7 bytes (Table 1 )

TABLE I. THE DESCRIPTION OF INITIAL 6 BYTES IN USER INFORMATION OF LONG SMS( TP-UD)

\begin{tabular}{|c|c|c|}
\hline Bytes & $\begin{array}{l}\text { Typical } \\
\text { value }\end{array}$ & Description \\
\hline 1 & 05 & Protocol header remaining bytes size: the protocol header of 6 byte is 05 while 06 for 7 byte \\
\hline 2 & 00 & $\begin{array}{l}\text { GSM 03. } 40 \text { regulation } 9.2 . \quad 3 . \quad 24 . \quad 1 \text { stipulates that } 00 \text { indicates } 1 \text { byte for the ID length } \\
\text { of long message }\end{array}$ \\
\hline 3 & 03 & Protocol header remaining bytes size \\
\hline 4 & 00 & The ID of long message is $00-$ FF. \\
\hline 5 & 05 & $\begin{array}{l}\text { The grouping number of the long message: } 05 \text { represents that this long message has } 5 \text { message } \\
\text { groups }\end{array}$ \\
\hline 6 & 01 & Present message group serial number: 01 presents that the present long message is the first group \\
\hline
\end{tabular}

III. Middleware Technology PrinciPle system software. It can connects systems to change

Middleware is an independent system software or service procedure while distributed application software takes advantages of it to share resources among diverse technologies. Middleware is located in the operation system of client/server o manage computer resource and internet communication, which connects two independent application procedures or independent information even when they have different interface. A key approach to implement middleware is information communication which can realize work among various platforms or in OS environment. 


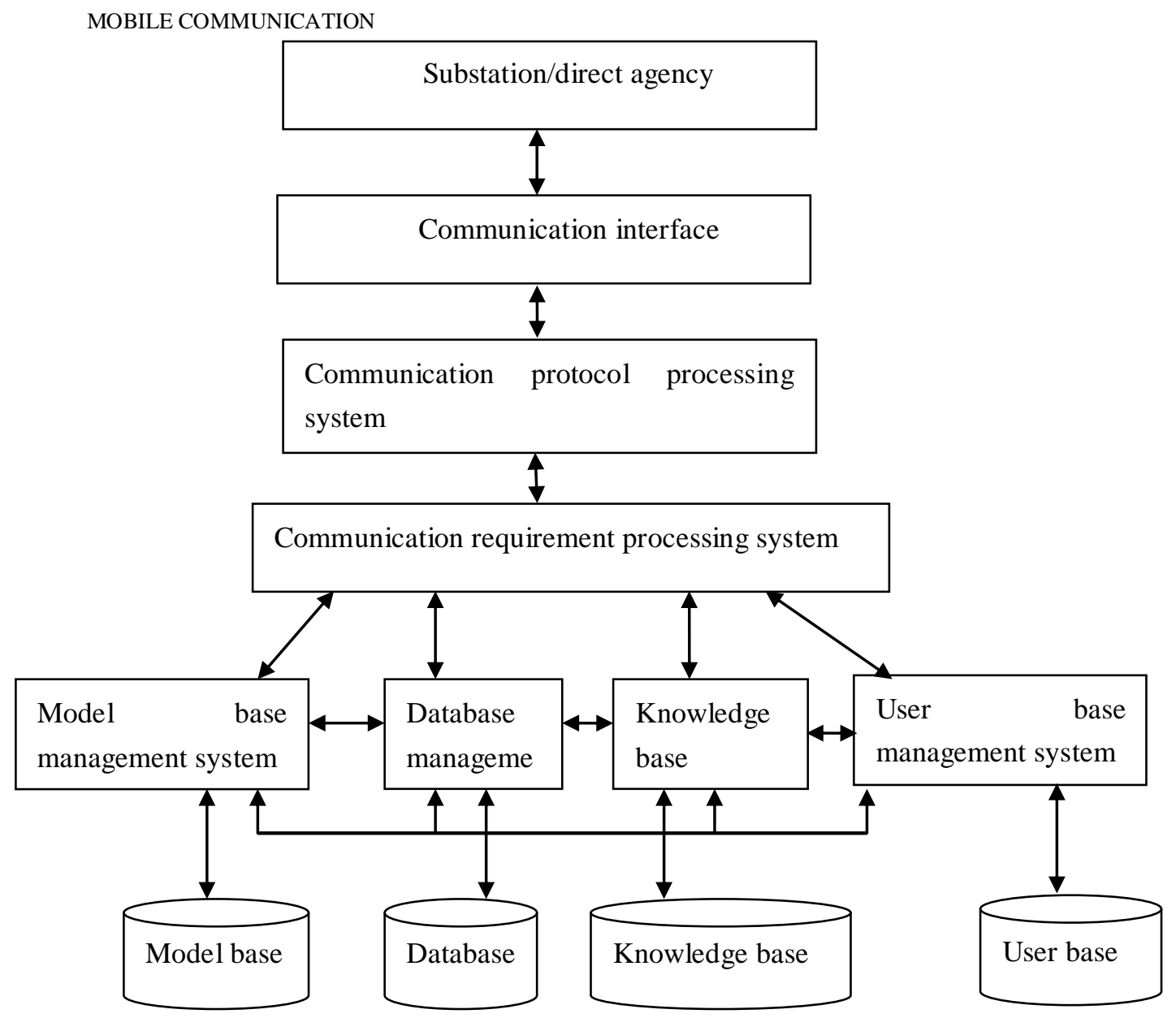

Figure 1.Mobile communication system structure

The communication data is responsible to process middleware to dismantle and reconstruct information data, verify the data order security and determine requirement, which dismantle the original data to other protocol data and send it to special processing server. It aims o realize the exchange between CMPP, telecom SMGP and Unicom SGIP so as to realize the data separation and analysis of HTTP, SOAP, TCP/IP protocol data and then generate new communication protocol.

This system deals with digital certificate releasing, verification and application. Besides, the middleware is adopted to carry out data capture, filtering, collection, computation, data check, demodulation, and deal with redundant data. Then, based on the data analysis result, it sends operation requirement to free electronic certificate releasing/validating server so as to reduce resource consumption, and meanwhile enhance bandwidth utilization.
At the same time, it provides special HTTP data interface protocol for current internal system which supports other interface modes.

\section{SYSTEM DESIGN}

The platform runs based on Linux operation system which adopts Java development and the database is Oracle. The platform uses SSH structure. The design obeys the following principle to carry out layer design which can be divided into three layers:

\section{A. Channel layer}

This layer is responsible to manage the channel and serve the whole system which sends and receives SMS/MMS, guaranteeing to effectively, timely and accurately deal with SMS/MMS.

\section{B. Business logic layer}

This layer aims to realize various business requirements and controls channel layer while provides data to it. 


\section{Interface layer}

External system interact with the information platform based on the interface provided by this layer.

1) Module independent principle

While creating the module, we have to guarantee its independence in terms of physical and logical perspectives.

2) Interface-oriented programming principles

In order to reduce the independence of various modules, we have to adopt interface-oriented programming principle so as to reduce the interconnection.

\section{3) Database design principle}

It takes database as the center, and the data change in information platform takes it as the media and assistive swap mode is SOCKET. Besides, necessary models have to be established so as to avoid confusion and a loss of efficiency to make efficiency reach the standard.

\section{CONCLUSION}

Mobile phones have almost replaced all portable electronic products, and smart phones have occupied very good position in the competition. This paper deeply analyzes the mobile communication multimedia information service system and turns it into real software products. The multimedia information service system is made up of short message service subsystem, MMS application subsystem, electronic certificate business application subsystem, streaming media service subsystem, and routing \& switching center system. What's more, core technologies solved in this paper include operator communication information acquisition technology, protocol analysis technology, and
SMS/MMS technology. In addition, mobile multimedia information system can provide service for government and SMS/MMS service for the public, such as make government affairs public, office service and satisfaction survey etc.

\section{REFERENCES}

[1] Onnela J P, Saramäki J, Hyvönen J, et al. Structure and tie strengths in mobile communication networks[J]. Proceedings of the National Academy of Sciences, 2007, 104(18):7332-7336.

[2] Castells, M., Fernez-Ardevol, M., Qiu, J. L., \& Sey, A. (2009). Mobile communication and society: a global perspective. Economic Geography, 53(1), 119-120.

[3] Manuel C, Mireia F, Qiu J L, et al. Mobile Communication and Society: A Global Perspective[J]. Economic Geography, 2008, 84(1):119-120.

[4] Anderson S, Millnert M, Viberg M, et al. An adaptive array for mobile communication systems[J]. Vehicular Technology IEEE Transactions on, 1991, 40(1):230-236.

[5] Patel C S, Pratt T G. Simulation of Rayleigh faded mobile-to-mobile communication channels[C]// Vehicular Technology Conference, 1988, IEEE 38th. 2003:1876 - 1884.

[6] Patel, Chirag S., and T. G. Pratt. "Simulation of Rayleigh-faded mobile-to-mobile communication channels." Communications IEEE Transactions on 53.11(2005):1876-1884.

[7] Fazel K. Performance of CDMA/OFDM for mobile communication system[C]// Universal Personal Communications, 1993. Personal Communications: Gateway to the 21st Century. Conference Record., 2nd International Conference on. IEEE, 1993:975-979 vol.2.

[8] Lambiotte R, Blondel V D, Kerchove C D, et al. Geographical dispersal of mobile communication networks $[\mathrm{J}]$. Physica A Statistical Mechanics \& Its Applications, 2008, 387(21):5317-5325.

[9] Stüber G L. Principles of Mobile Communication," 2nd ed[J]. Transmitter Array Receiver Array Spatial Multipath Channel Scatterers J T J R J T J T J T J T J R J R H H H H, 2001.

[10] Shimizu K. Telemedicine by Mobile Communication[J]. IEEE Engineering in Medicine \& Biology Magazine, 1999, 18(4):32 44.

[11] Tsoulos G V. Smart antennas for mobile communication systems: benefits and challenges[J]. Electronics \& Communications Engineering Journal, 1999, 11(2):84-94.

[12] Mccormick A C, Al-Susa E A. Multicarrier CDMA for future generation mobile communication[J]. Electronics \& Communication Engineering Journal, 2002, 14(2):52-60. 